\title{
Visuospatial Perception in Children Born Preterm With No Major Neurological Disorders
}

\author{
Phillipa R. Butcher \\ University of Groningen and Australian National University
}

\author{
Elisabeth F. Stremmelaar and Arend F. Bos \\ University Medical Centre Groningen, Groningen, The \\ Netherlands
}

\author{
Anke Bouma \\ University of Groningen \\ Michael Smithson \\ Australian National University
}

Koenraad N. J. A. Van Braeckel

University of Groningen and University Medical Centre Groningen, Groningen, The Netherlands

\begin{abstract}
Objective: Many investigations have found deficits in visuospatial perception in children born preterm, however, it is not clear whether the deficits are specific to visuospatial perception or the consequences of deficits in other functional areas, which often accompany preterm birth. This study investigated whether children born preterm show a specific deficit in visuospatial perception. Method: Fifty-six 7- to 11 -year-old preterm born children (gestational age $<34$ weeks) without cerebral palsy and 51 agematched, full-term children completed four computerized tasks tapping different levels and types of visuospatial perception. Accuracy and speed of responses were recorded. Task formats were designed to reduce demands on attentional deployment. Measures of intelligence and parental education were included in the analysis. Results: Children born preterm performed less accurately and/or less rapidly on all tasks. Their poorer performance did not reflect differences in speed-accuracy trade-off. Parental education and IQ, both significantly lower in the preterm children, contributed positively to performance on all tasks. IQ mediated the association between preterm birth and visuospatial performance on the most cognitively demanding task. Conclusion: Children born preterm performed more poorly than full-term controls on four visuospatial perceptual tasks. Although intelligence and parental education were also associated with performance, preterm birth contributed independently of these factors on three of four tasks. Many children born preterm are thus multiply disadvantaged on visuospatial tasks: the lower IQ scores and parental educational levels frequently found in this group increase the deficit associated with preterm birth.
\end{abstract}

Keywords: preterm, visuospatial perception, dorsal stream vulnerability

Visuospatial perceptual deficits in children born preterm $(<37$ weeks gestational age [GA]) have been a subject of investigation for more than twenty years. Some studies have used tasks that tap specific aspects of visuospatial processing, such as perception of line orientation or Gestalt closure (Atkinson \& Braddick, 2007; Breslau, Chilcoat, DelDotto, \& Andreski, 1996; Foreman, Fielder, Minshell, \& Hurrion, 1997; Jakobson, Frisk, Knight, Downie, \& Whyte, 2001; Jakobson, Frisk, \& Downie, 2006; O'Reilly et al., 2010; Santos, Duret, Mancini, Busuttil, \& Deruelle, 2010; Taylor, Minich, Klein, \& Hack, 2004; Taylor, Jakobson, Maurer, \& Lewis,
2009). Other studies have used scores from test batteries designed to provide a more global assessment of visuospatial processing such as the Test of Visual Perceptual Skill (TVPS) or the Motor Free Visual Perception Test (MVPT) (Caravale, Tozzi, Albino, \& Vicari, 2005; Davis, Burns, Wilkerson, \& Steichen, 2005; Goyen, Lui, \& Woods, 1998; Hård, Niklasson, Svensson, \& Hellström, 2000; McGrath \& Sullivan, 2002; Vicari, Caravale, Carlesimo, Casadei, \& Allemand, 2004; O'Reilly et al., 2010; Teplin, Burchinal, Johnson-Martin, Humphry, \& Kraybill, 1991). The majority of these studies have found that children born preterm perform worse
This article was published Online First August 20, 2012

Phillipa R. Butcher, Clinical and Developmental Neuropsychology, University of Groningen, Groningen, The Netherlands, and Department of Psychology, Australian National University, Canberra, Australia; Anke Bouma, Clinical and Developmental Neuropsychology, University of Groningen, Groningen, The Netherlands; Elisabeth F. Stremmelaar and Arend F. Bos, Department of Pediatrics, University Medical Centre Groningen, Groningen, The Netherlands; Michael Smithson, Department of Psychology, Australian National University, Canberra, Australia; and Koenraad N. J. A. Van Braeckel, Clinical and Developmental Neuropsychology,
University of Groningen, Groningen, The Netherlands, and Department of Pediatrics, University Medical Centre Groningen, Groningen, The Netherlands.

This research was made possible by grants from the School of Behavioral and Cognitive Neurosciences, BCN of University of Groningen, and the Netherlands Organization for Scientific Research, NWO.

Correspondence concerning this article should be addressed to Phillipa R. Butcher, Department of Psychology (Building 39), Australian Nationa University, Canberra, ACT 0200, Australia. E-mail: Phillipa.Butcher@ anu.edu.au 
than their full-term age-mates in some or all of the areas tested (Atkinson \& Braddick, 2007; Breslau et al., 1996; Caravale et al., 2005; Davis et al., 2005; Hård et al., 2000; Klein, 1988; Luoma, Herrgård, \& Martikainen, 1998; McGrath \& Sullivan, 2002; Santos et al., 2010). Although participants in one study, which found deficits were extremely preterm $(\mathrm{GA} \leq 28$ weeks) with established neurological impairment (Hård et al., 2000), participants in the remaining studies were preterm $(\mathrm{GA} \leq 36$ weeks: Caravale et al., 2005; Vicari et al., 2005) or very preterm born children (GA $\leq 32$ weeks: Atkinson \& Braddick, 2007; Breslau et al., 1996; Davis et al., 2005; Foreman et al., 1997; Klein, 1988; Luoma et al., 1998; Santos et al., 2010) with no major neurological impairment.

Although this suggests that visuospatial perception may be particularly vulnerable to the disruption in development associated with preterm birth, several studies of very preterm children, including some participants with extremely low birth weight (ELBW), did not find deficits (very preterm: Jakobson et al., 2006; O'Reilly et al., 2010; Taylor et al., 2004; Taylor et al., 2009; ELBW: Goyen et al., 1998; Jakobson et al., 2001; Teplin et al., 1991). Further, studies that found deficits had methodological limitations, which make it unclear whether the deficits identified were specific to visuospatial perception. For example, most tests of perception call on a range of skills, including spatial insight, analytical skill, and strategic thinking, with the result that both perceptual and intellectual skills contribute to performance (O'Reilly et al., 2010; Stiers, De Cock, \& Vandenbussche, 1999; Trojano et al., 2004). Although most previous studies excluded children with IQs below 70, few took possible associations between IQ and performance into account in their analyses (see Caravale et al., 2005; Davis et al., 2005, for exceptions). The deficits identified may therefore have been associated with the depressed intellectual skills consistently found in children born preterm (Bhutta, Cleves, Casey, Cradock, \& Anand, 2002), making it unclear whether they were deficits in perceptual skill, intellectual skill, or a combination of both.

A second limitation is that many tests place high demands on attentional regulation and inhibitory control. TVPS and MVPT matching tasks, for example, require the ability to scan systematically four or five highly similar patterns, withholding responses to distracters, until the correct pattern has been identified. Children born preterm are more likely than full-term age-mates to have difficulty with the attentional control, which these tasks demand (Mulder, Pitchford, Hagger, \& Marlow, 2009; Pizzo et al., 2010). This makes it difficult to determine to what extent incorrect responses reflect differences in the regulation of attention rather than in visuospatial perception.

A final limitation is that most existing studies reported only the accuracy of responses (see Foreman et al., 1997, for an exception). As such, they have provided a measure of effectiveness-how well children perform, measured in terms of the correctness of their responses-but not of efficiency-how much effort they must expend to perform effectively, measured in terms of time taken to arrive at a correct response (Eysenck, Derakshan, Santos, $\&$ Calvo, 2007). Both aspects of performance must be considered to determine whether deficits in visuospatial perception exist. Failure to control for the intelligence of the child or to take the attentional demands of the task into account may overestimate the extent of any visuospatial perceptual deficits; relying solely on measures of accuracy may underestimate it.
The goal of our study was to determine whether children born preterm show specific deficits in visuospatial perception. Visuospatial perception refers broadly to the analysis of spatial relationships among different objects or the component elements of a single object (Trojano et al., 2004). Visual information is processed hierarchically, and visuospatial perception ranges in complexity from elementary processes involved in the very early stages of visual analysis, such as detection of orientation, or location, to higher level processes, which require the spatial integration of the features or components of a stimulus and the formation and manipulation of mental representations (Trojano et al., 2004). Visuospatial perceptual processes differ not only in their complexity, but also in their spatial frame of reference. Two broad visual streams, each associated with a specific neural pathway, have been distinguished on this basis (Mishkin, Ungerleider, \& Macko, 1983; Milner \& Goodale, 2006). The ventral pathway, which projects ventrally from the primary visual cortex in the occipital lobe to the inferotemporal cortex, processes the spatial relationships required to represent the external world using allocentric coding, which permits recognition and classification across a range of viewpoints (Creem \& Proffitt, 2001; Fias, Dupont, Reynvoet, \& Orban, 2002). The dorsal pathway, which projects dorsally from the primary visual cortex to the posterior parietal cortex, processes the spatial relationships required for visually guided action such as reaching and grasping, using egocentric reference systems, and for the perception of motion, distance, and location, using environmental reference systems (Creem \& Proffitt, 2001; Fias et al., 2002).

Children born very preterm have been found to be at particularly high risk for deficits in functions mediated by the dorsal visual pathway (Atkinson \& Braddick, 2007; Foreman et al., 1997; Santos et al., 2010; Taylor et al., 2009). The dorsal pathway flows through the periventricular area, site of the white matter injury that is the most common form of neural pathology in very preterm infants (Volpe, 2009). Very early periventricular white matter injury is frequently associated with short- and long-term disturbances in myelination (Nosarti et al., 2002), decreased white and gray matter volume at term age (Inder, Warfield, Wang, Hüppi, \& Volpe, 2005; Ment, Hirtz, \& Hüppi, 2009), and structural anomalies in childhood and adolescence. Reduced gray matter volumes have been found in occipital (Nosarti et al., 2002) and parietooccipital regions (Peterson et al., 2000), and white matter abnormalities in occipital and parietal lobes in children and adolescents born preterm (Giménez et al., 2006; Nosarti et al., 2002). However, structural anomalies in preterm children are not restricted to dorsal stream areas. Temporal and frontal cortex also show reductions in gray matter (Nosarti et al., 2002; Peterson et al., 2000), and alterations in white matter (Nosarti et al., 2002), while subcortical structures, including the thalamus, basal ganglia, and cerebellum, may also show structural alterations (Ment et al., 2009; Volpe, 2009). The widespread nature of these differences raises the possibility that visuospatial functions mediated by other pathways may also be impaired. Consistent with this, previous research has also found deficits on tasks not generally associated with the dorsal stream, for example, TVPS subtests (Davis et al., 2005), shape matching (Atkinson \& Braddick, 2007), configural processing (Santos et al., 2010), and global form perception (Taylor et al., 2009). 
To determine which aspects of visuospatial perception were impaired, we compared the performance of children born preterm and full term on four tasks tapping different types of visuospatial processing. One task required a quantitative judgment of spatial location, which is generally associated with the dorsal stream (Chen, Myerson, Hale, \& Simon, 2000; Fias et al., 2002). The remaining three tasks required the combined functioning of dorsal and ventral streams. One assessed the ability to match line orientations, which, while calling on areas of the dorsal stream $(\mathrm{Ng}$ et al., 2000; Tibber, Anderson, Melmoth, Rees, \& Morgan, 2009), has also been associated with the ventral extrastriate cortex (Isaacs, Edmonds, Chong, Lucas, \& Gadian, 2003; Ng et al., 2001). Estimating distance and matching line orientation were considered to address elementary perceptual processes. Two tasks were considered to address higher-level visuospatial processing: matching complete and incomplete geometric patterns. These tasks required judgments of identity based on analyzing and forming representations of complex spatial configurations. Such tasks call on the integration of perceptual and cognitive processes, which involve a range of areas in both dorsal and ventral streams (Cohen, Dehaene, Vinckier, Jobert, \& Montavont, 2008; Zaehle et al., 2007). The revised MVPT (MVPT-R), a test of similarly complex visuospatial perception, for example, has been found to activate a broad network of areas, including primary visual, visual association, as well as parietal and frontal cortices (Calhoun et al., 2001).

To determine whether any deficits so identified were specific to visuospatial processing, factors identified in previous research as possible confounders were taken into account in the task design or the analysis. First, measures of intelligence were included in the analyses. Because verbal and nonverbal intelligence tap different aspects of the global intellectual skills, which may be called on by visuospatial tasks (Isaacs et al., 2000), measures of both were included. Second, to reduce demands on inhibitory control made by multiple distracters, all visuospatial tasks used forced-choice formats with a single distracter. Third, both accuracy and latency of responses were investigated. Finally, number of years of parental education was included in the analysis as an index of the quality of the home environment, which has been found to have a pervasive effect on development and which may differ systematically between preterm and full-term children (Laucht, Esser, \& Schmidt, 1997).

Given existing evidence for deficits on functions associated with the dorsal visual stream, we expected that, after controlling for intelligence and parental education, children born preterm would judge distance less accurately and less rapidly. The finding of lower accuracy or longer reaction times (RTs) on one or more of the remaining tasks would provide evidence for the existence of more broadly based visuospatial deficits.

\section{Method}

\section{Participants}

Fifty-six preterm born children (30 boys; $M_{\mathrm{GA}}=29$ weeks, 5 days; range: 25 weeks, 5 days -33 weeks, 5 days; $M_{\text {birth weight }}=$ $1188 \mathrm{~g}$; range: $595-1800 \mathrm{~g}$ ) between 7 and 11 years of age took part in the study. Seventy of the children admitted within 24 hours of birth to the neonatal intensive care unit (NICU) of the University Medical Centre Groningen (UMCG) between October 1992 and January 1996 met the inclusion criteria for the study: preterm birth, no chromosomal abnormalities, congenital malformations, cerebral palsy, or visual or auditory sensory loss. Five children could not be located at the time of the study. Nine declined to participate. The 56 participants $(80 \%$ of those eligible to participate) can be considered typical of preterm infants admitted in the mid 1990s to the NICU of UMCG who did not develop severe neurological disorders. Although 50 children (90\%) met the criteria for very preterm birth ( $\mathrm{GA} \leq 32$ weeks), six $(10 \%)$ were born at $<34$ weeks GA. Eleven $(20 \%)$ were small for GA $(<5$ th percentile). Perinatal clinical characteristics are presented in Table 1. All

Table 1

Perinatal Clinical Characteristics of the Preterm Group

\begin{tabular}{lc}
\hline \multicolumn{1}{c}{ Characteristic } & $M$ (range) or $n / N(\%)$ \\
\hline Gestational age & 29 weeks, 5 days $(25$ weeks, 5 days-33 weeks, 5 days $)$ \\
Birth weight & $1188 \mathrm{~g}(595-1800 \mathrm{~g})$ \\
SGA (birth weight $<5$ th percentile) & $11 / 56(20)$ \\
Prenatal corticosteroids & $40 / 56(71)$ \\
IPPV & $28 / 56(50)$ \\
Septicemia & $20 / 56(36)$ \\
ICH grade 1-2 & $12 / 56(21)$ \\
ICH grade 3-4 & None \\
PVL grade 1 & $28 / 56(50)$ \\
PVL grade 2-3 & None \\
NBRS at term age & $3(0-7)$ \\
BPD & $11 / 56(20)$ \\
Postnatal corticosteroids & $7 / 56(12.5)$ \\
Retinopathy of prematurity & None \\
\hline
\end{tabular}

Note. $\quad$ SGA $=$ small for gestational age, according to Dutch weight centiles of Kloosterman (1970); IPPV = intermittent positive pressure ventilation; ICH = intracranial hemorrhage, as graded according to Papile et al. (1978); PVL = periventricular leukomalacia, as graded according to de Vries, Ekers, \& Dubowitz, (1992); NBRS = nursery neurobiologic risk score, a neonatal risk score (Brazy, Eckerman, Oehler, Goldstein, \& O'Rand, 1991); BPD = bronchopulmonary dysplasia, defined as oxygen dependency at 36 weeks postmenstrual age. 
children had corrected vision when required. No child met the World Health Organization criteria for low vision $(<10$ cycles per degree of visual angle; http://www.who.int/blindness/causes/ priority/en/index 5.html).

The control group comprised 51 full-term children (28 boys; $M_{\mathrm{GA}}=40$ weeks, 1 day; range: $37-42$ weeks); $M_{\text {birth weight }}=$ $3637 \mathrm{~g}$; range: $2580-4949 \mathrm{~g}$ ) between 7 and 11 years of age with uneventful pre- and perinatal histories and no known developmental disabilities. The children had accepted an invitation to participate in the study sent out through their mainstream elementary school. Full-term participants were closely, but not completely, matched to the preterm participants in terms of sex and age (one fewer 7-year-old girl; four fewer 9-year-old boys). They were similar to the general population on Movement Assessment Battery for Children scores (M-ABC, Smits-Engelsman, 1998): children with a Total M-ABC score or a Fine Motor score $\leq 5$ th percentile were excluded. Mean Total M-ABC score was 45th percentile (range: $8-92$ percentile). The study and all procedures were approved by the ethical review board of UMCG.

\section{Procedure}

Data were collected as part of a longitudinal study of the development of children born preterm. The parent(s) and their child(ren) attended a test session at UMCG where the children's intelligence, motor skill, neurological, and medical status were assessed. Approximately fourteen months later $(S D=2$; range: 11-17), parents and children attended an experimental session at the University of Groningen, where the children carried out a series of tasks measuring visuospatial processing skills.

Each child was seated in front of a computer screen tilted at an angle of $15^{\circ}$ from the horizontal. The borders of the screen and the table on which it rested matched the color of the screen. The height of the chair could be adjusted so the child's forearm rested comfortably on a support while the index fingers rested on response keys located between child and screen. The child's face and the display on the screen were shown on a super VHS video monitor, allowing one researcher to present the experimental display when the child was attending to the screen. A second researcher stood behind the child, monitoring posture and encouraging concentra- tion. The child initiated each trial by pressing a key triggering the appearance of a colored marble at a central fixation point along the midline of the screen. Between 750 and 3000 ms after the child fixated on the marble, the display was presented. Practice sessions of five to six trials, lengthened where necessary to ensure understanding, preceded each task.

\section{Measures}

Background characteristics. Intelligence was assessed using four Verbal (VIQ) subtests (Similarities, Arithmetic, Vocabulary, Comprehension) and three Performance (PIQ) subtests (Picture Arrangement, Block Design, Object Assembly) from the WISC III ${ }^{\mathrm{NL}}$ (Kort et al., 2002). VIQ and PIQ scores were calculated using Sattler's (1992) formula. Scores are presented in Table 2.

Visuospatial perception. Responses were recorded by a key press. This provided measures of both accuracy and RT, and allowed for inferences about efficiency and speed-accuracy tradeoff to be drawn.

Judgment of distance. This task was based on the dot location task described in Chen et al. (2000). Two orange spots were presented $10 \mathrm{~cm}$ apart, along an axis $20^{\circ}$ from the midline on the side of the dominant hand. After $800-900 \mathrm{~ms}$, a green spot appeared on or close to the same axis between the orange spots. The distance from the green spot to the nearer orange spot was varied to provide trials, which differed systematically in difficulty, with spots located $2.75,2.00,1.25$ or $.500 \mathrm{~cm}$ from the point midway between the two orange spots. See sample trials in panel 1 of Figure 1. There were six trials at each difficulty level. Right-handed children were informed that the left key represented the nearer (left) orange spot, while the right key represented the further (right) orange spot. The situation was reversed for lefthanders. The children were asked to press the key representing the orange spot nearer to the green spot. The task took approximately five minutes.

Matching line orientation. This task was based on the Judgment of Line Orientation Test (Benton, Hamsher, Varney, \& Spreen, 1983). Two lines, each $4 \mathrm{~cm}$ long and $1 \mathrm{~mm}$ wide, were presented on the top half of the screen. Their lower, inner edges were $1 \mathrm{~cm}$ apart, equidistant from the vertical midline of the

Table 2

Background Characteristics of the Full-Term and Preterm Born Children

\begin{tabular}{|c|c|c|c|}
\hline \multirow[b]{2}{*}{ Characteristic } & \multicolumn{2}{|c|}{ Group } & \multirow[b]{2}{*}{$t(d f)$ or $\chi^{2}$} \\
\hline & Full term & Preterm & \\
\hline \multicolumn{4}{|l|}{ Age } \\
\hline Session 1 (years:months) & $8: 8(S D$ 1:1), range: $7: 2-10: 6$ & $8: 6(S D$ 1:0), range: $7: 1-10: 5$ & $.809(105)$ \\
\hline Session 2 (years:months) & $10: 1(S D$ 1:2), range: $7: 3-11: 9$ & $10: 2(S D$ 1:2), range: $7: 3-11: 9$ & .109 (105) \\
\hline Gender (male/female) & $28 / 23$ & $30 / 26$ & $.75(1)$ \\
\hline Parents' education ${ }^{\mathrm{a}}$ & 3.5 (SD .94), range: $0-5$ & $2.6(S D .88)$, range: $1-5$ & \\
\hline VIQ & $108(S D$ 10.1), range: $81-126$ & 93 (SD 12.7), range: $68-125$ & $6.69(105)^{* * * * *}$ \\
\hline PIQ & $104(S D$ 12), range: $77-133$ & 95 (SD 12.9), range: $66-119$ & $3.73(105)^{* * * *}$ \\
\hline
\end{tabular}

Note. Data: mean (standard deviation), range or $n / n$.

a Parents' education was coded as follows:

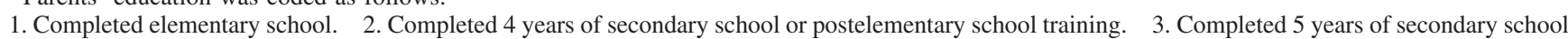
or 4 years of secondary school followed by occupational training. 4. Completed nonacademic tertiary education. 5. Completed academic tertiary education. 


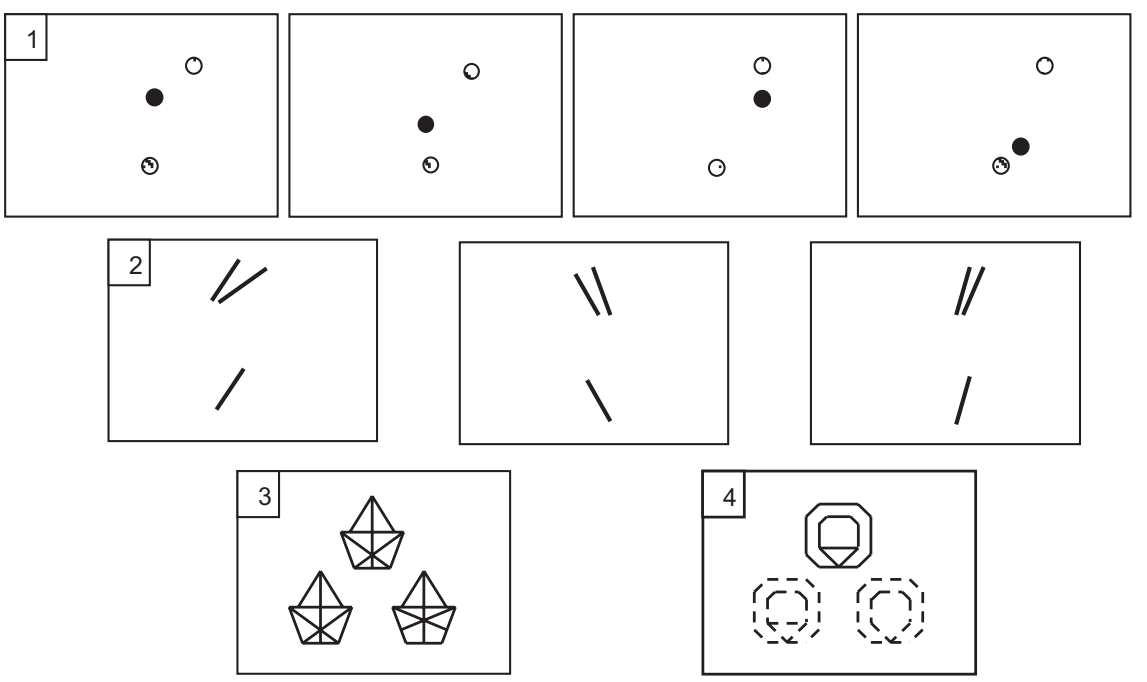

Figure 1. Examples of stimuli from the four tasks: Task 1. Distance (distance from closer dot, from left to right: 4.5, 3.75, 3.00, and $2.25 \mathrm{~cm}$ ); Task 2: Line orientation (size of angle, from left to right 27, 18, and $9^{\circ}$ ); Task 3 . Patterns; and Task 4. Incomplete patterns. The two upper panels represent the two tasks that call on elementary perceptual functions. The lower panel represents the two tasks that call on higher-order perceptual functions.s

screen. The pairs of lines subtended angles of $27^{\circ}$ (eight trials), $18^{\circ}$ (eight trials), or $9^{\circ}$ (eight trials), providing trials differing systematically in difficulty. See sample trials in panel 2 of Figure 1. After $800-900 \mathrm{~ms}$, a line parallel to one of the top lines appeared in the lower half of the screen, across the vertical midline. Trials were ordered so that angles of the same size were not presented consecutively on the same side, and target lines of the same orientation were not presented consecutively. Children were informed that the left key represented the upper left line and the right key represented the upper right line, and they were asked to press the key representing the line that pointed in the same direction as the lower line. The task took approximately five minutes.

Matching geometric patterns. This task was based on the Visual Discrimination subtest of the revised TVPS (TVPS-R; Gardner, 1996). A single geometric pattern was displayed on the top half of the screen. Two similar patterns, one identical to the top pattern, were displayed on the lower half of the screen. See the sample trial in panel 3 of Figure 1. The display remained for $10 \mathrm{~s}$. Sixteen trials were presented, with correct responses distributed equally across left and right hands. Stimuli were presented in the same order as in the Visual Discrimination subtest. However, only one distracter was presented: the incorrect alternative selected most frequently in a pilot study using the pen-and-pencil version of the TVPS-R with typically developing 7 - to 10 -year-old children. Children were informed that the left key represented the pattern on the left lower half of the screen while the right key represented the pattern on the right lower half of the screen, and they were asked to press the key corresponding to the pattern on the lower half of the screen, which was identical to the top pattern. The task took approximately three minutes.

Matching incomplete geometric patterns. This task was based on the Visual Closure subtest of the TVPS-R (Gardner, 1996). The task was identical to geometric patterns except that the patterns displayed on the lower half of the screen were incomplete. See the sample trial in panel 4 of Figure 1. As with the geometric patterns, 16 trials were presented, using as a distracter the incorrect alternative selected most frequently in a pilot study using the pen-and-pencil version of the TVPS-R with typically developing children.

\section{Analysis}

Trials on which the child was inattentive or not sitting appropriately were excluded from the analyses. Anticipatory responses ( $\leq 200 \mathrm{~ms}$ on distance, $\leq 250 \mathrm{~ms}$ on line orientation as well as matching and incomplete geometric patterns) and responses more than $3 S D$ above the child's mean RT were also excluded. In the preterm group, $12(0.8 \%), 25(1.8 \%), 23(2.4 \%)$, and 16 trials (1.6\%) were dropped on distance, line orientation, matching patterns, and incomplete patterns, respectively. The corresponding figures for the control group were $11(0.7 \%), 34(2.4 \%), 14$ $(1.7 \%)$, and 12 trials $(1.5 \%)$.

Responses on individual items of the tasks were nested within children, violating the assumption of independence in analysis of variance-based methods. Associations between birth status (preterm vs. full term) and performance were therefore analyzed using multilevel modeling (SPSS, Version 16, for the RT data, Stata for the frequency of correct responses). Multilevel analysis has the further advantage of allowing unequal numbers of observations per participant. Unequal numbers occurred in the analysis of correct responses when trials on which the child was inattentive, anticipated, or responded too slowly were excluded, and in the analysis of the RTs, when incorrect responses were excluded.

RTs on all tasks were positively skewed. Analyses were therefore carried out on logarithmic transformations. Two-level hierarchical models were used in the analyses of matching patterns and incomplete patterns. First-level units were the items; second-level units were the participants. Three-level hierarchical models were used for the analysis of distance and line orientation. First, second- 
and third-level units were, respectively, the items, the difficulty conditions, and the participants.

Age, gender, and parents' education were expected to influence performance on the visuospatial tasks. They were therefore entered together with birth status group in the first step in all models. Male sex is considered an added risk for neurodevelopmental deficits in children born preterm (Constable et al., 2008; Kesler et al., 2008), and developmental trajectories in children born preterm and full term may differ (Gozzo et al., 2009). Interactions between group, sex, and age were therefore also entered at this stage. At Step 2, IQ scores were entered. VIQ and PIQ may not be equally impaired in children born preterm-PIQ is frequently lower than VIQ (Isaacs et al., 2000). Further, subtests that make up PIQ call on visuospatial perceptual skills while subtests that make up VIQ call on verbal skills (Taylor et al., 2009). To tease out the contributions of the different types of intellectual skill, the analysis was conducted with VIQ and PIQ rather than Full-scale IQ (FSIQ). Because the associations between intelligence and visuospatial perception may be altered by the disturbances that frequently affect brain development in children born preterm (Kesler et al., 2008; Peterson et al., 2000), interactions between IQ and group were also entered.

\section{Results}

The children born preterm scored significantly lower than the controls on VIQ and PIQ, $t(105)=6.69, p<.001 ; t(105)=3.73$, $p<.001$, respectively (see Table 2). VIQ and PIQ correlated significantly in both groups (control: $r=.29, p=.04$; preterm: $r=.59, p<.001)$. Parents of children born preterm had significantly fewer years of education than parents of full-term children, $t(102)=5.15, p<.001$ (see Table 2$)$.

\section{Associations Between Birth Status and Performance on Measures of Visuospatial Ability}

Mean scores on the experimental tasks in the two groups are presented in Table 3 . The results of the analyses are presented in Tables 4 and 5.

\section{Distance}

After controlling for the covariates age, gender, and parents' education, there were no significant differences in accuracy between the two groups $(p<.05)$. Preterms' responses were significantly slower, $F(1,107)=7.79, p=.006$. RTs increased in both groups as difficulty increased, $F(3,345)=71.01, p<.001$, however, they increased significantly more in the preterm group in the most difficult condition, $t(320) 2.71, p=.007$. No other interactions were significant.

At Step 2a, RTs decreased significantly as VIQ increased, $F(1$, $109)=6.48, p=.012$. The contribution of group and the interaction between group and difficulty remained significant $(p=.04$, $p=.004$, respectively). At Step 2b, RTs decreased significantly as PIQ increased, $F(1,107)=12.38, p=.001$. The contribution of

Table 3

Accuracy and Speed of Responding on Tasks Measuring Visuospatial Perception for the FullTerm and Preterm Born Children

\begin{tabular}{|c|c|c|c|}
\hline \multirow[b]{2}{*}{ Task } & \multicolumn{2}{|c|}{ Group } & \multirow[b]{2}{*}{$\begin{array}{c}\text { Relative deficit in accuracy } \\
\text { or speed }(\%)\end{array}$} \\
\hline & $\begin{array}{l}\text { Full term } \\
M(S D)\end{array}$ & $\begin{array}{l}\text { Preterm } \\
M(S D)\end{array}$ & \\
\hline \multicolumn{4}{|l|}{ Distance } \\
\hline \multicolumn{4}{|l|}{ Accuracy (\%) } \\
\hline Difficulty: low & $99(4)$ & $99(5)$ & 0 \\
\hline Difficulty: medium & $99(4)$ & $99(5)$ & 0 \\
\hline Difficulty: high & $98(5)$ & $96(8)$ & 98 \\
\hline \multicolumn{4}{|l|}{ Reaction time (s) } \\
\hline Difficulty: low & $740(207)$ & $910(470)$ & $81^{* * * *}$ \\
\hline Difficulty: medium & $840(380)^{* * *}$ & $980(470)$ & $92^{* *}$ \\
\hline Difficulty: high & $1210(830)$ & $1730(1380)$ & $70^{\text {****** }}$ \\
\hline \multicolumn{4}{|l|}{ Line orientation } \\
\hline \multicolumn{4}{|l|}{ Accuracy (\%) } \\
\hline Difficulty: low & $97(9)$ & $88(20)$ & $91^{* *}$ \\
\hline Difficulty: medium & $94(11)$ & $84(22)$ & $89^{* * *}$ \\
\hline Difficulty: high & $83(17)$ & 84 (18) & 98 \\
\hline \multicolumn{4}{|l|}{ Reaction time (s) } \\
\hline Difficulty: low & $1680(990)$ & $1860(1050)$ & 90 \\
\hline Difficulty: medium & $1910(1190)$ & $2100(1230)$ & 91 \\
\hline Difficulty: high & $2630(1710)$ & $2700(1750)$ & 97 \\
\hline \multicolumn{4}{|l|}{ Matching patterns } \\
\hline Accuracy $(\%)$ & $89(10)$ & $81(13)$ & $91^{* * *}$ \\
\hline Reaction time (s) & $2730(2090)$ & $3350(2090)$ & $81^{* *}$ \\
\hline \multicolumn{4}{|l|}{ Incomplete patterns } \\
\hline Accuracy (\%) & $82(12)$ & $75(12)$ & $91^{* * *}$ \\
\hline Reaction time (s) & $3290(2450)$ & $3720(2320)$ & $88^{\text {******* }}$ \\
\hline
\end{tabular}

Note. Relative deficit expresses the preterm performance as a percentage of the full-term performance.

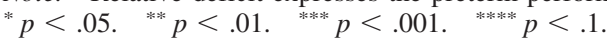




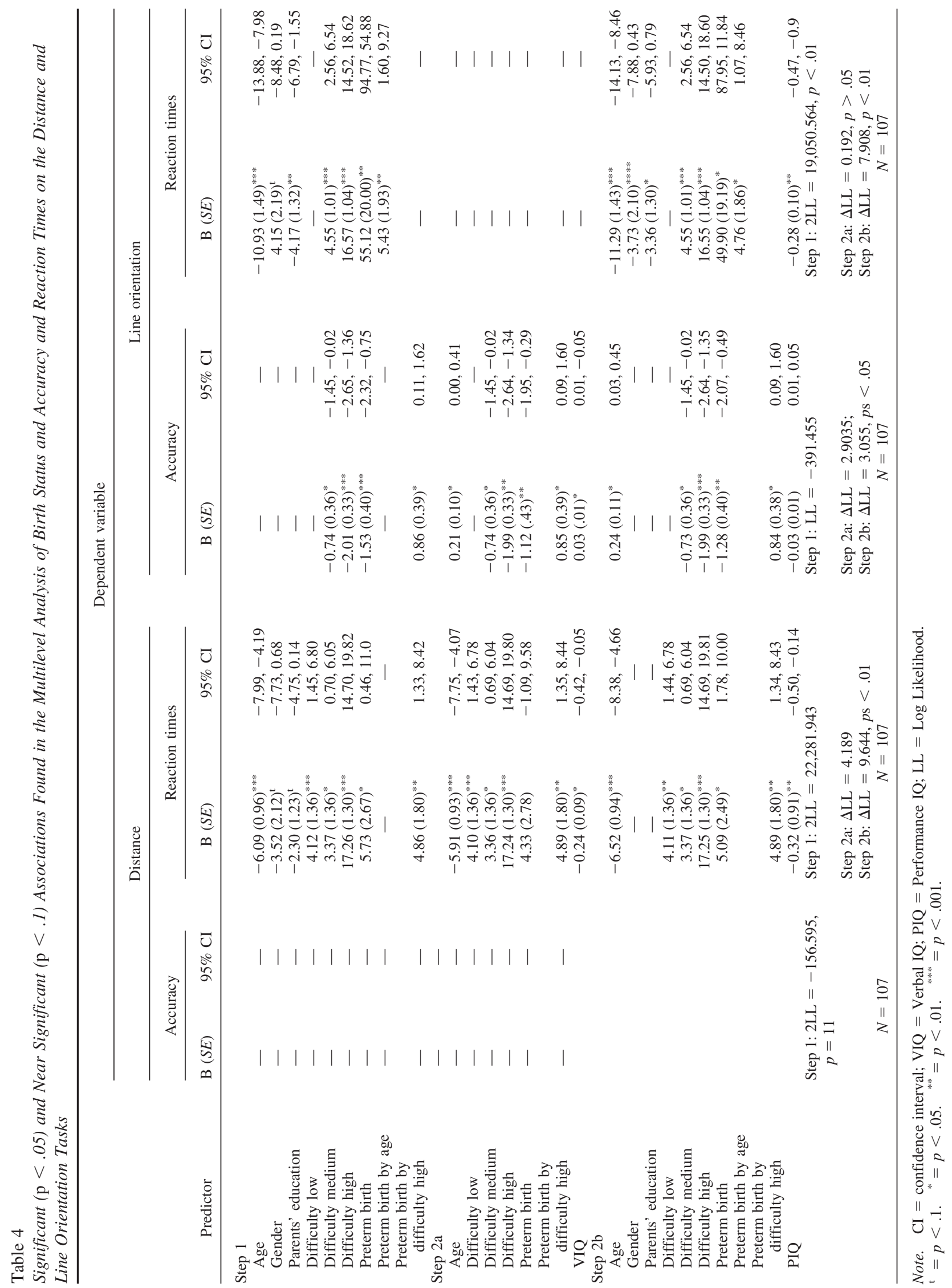


BUTCHER ET AL.

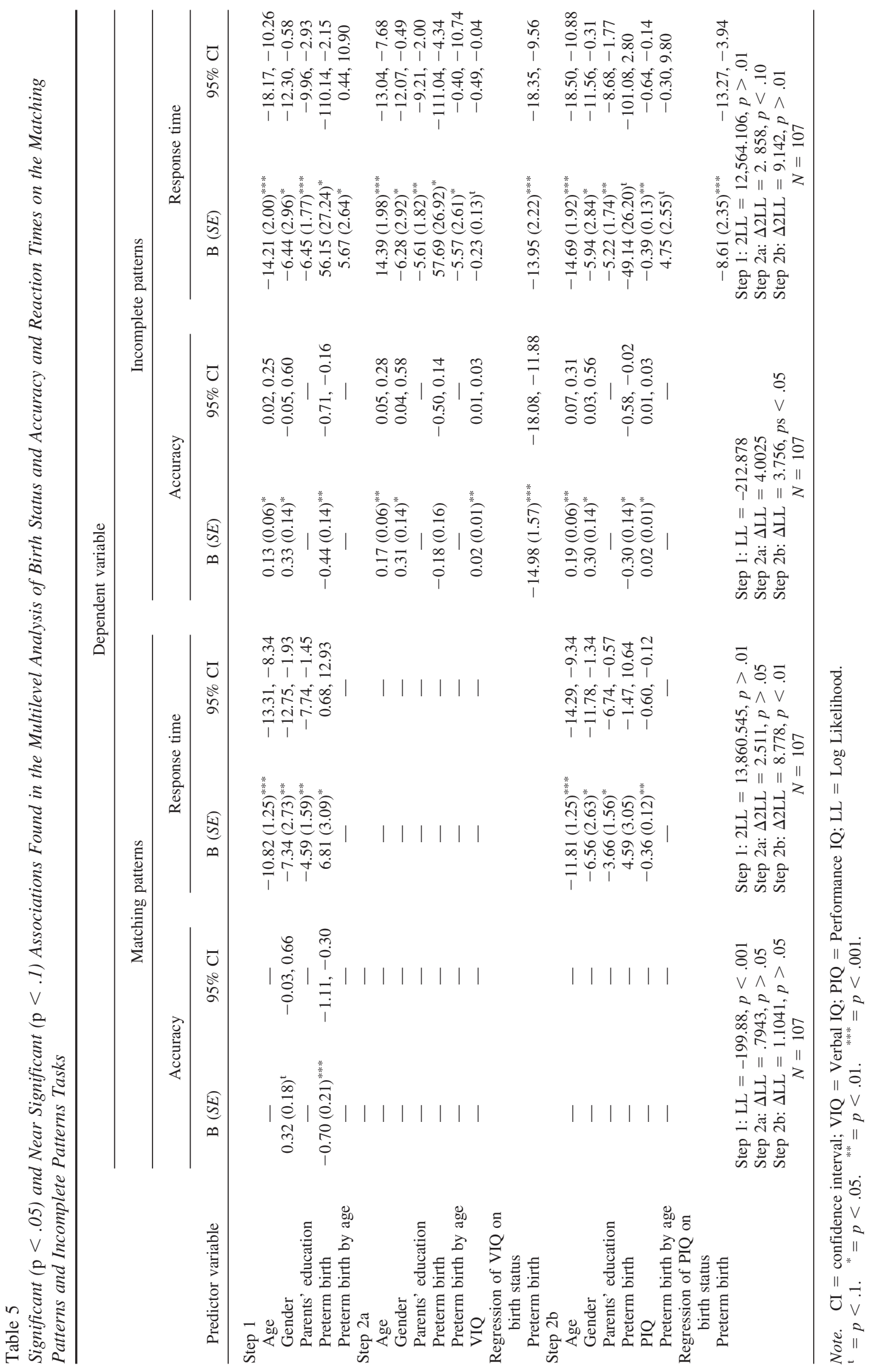


group and the interaction between group and difficulty remained significant ( $p=.007, p=.004$, respectively). The interactions between VIQ, PIQ, and group were not significant $(p>.05)$.

\section{Line Orientation}

After controlling for the key covariates, the preterm children were significantly less accurate than the control group, $z=-3.84, p<$ .001. Accuracy decreased as difficulty increased in both groups, $z=$ $-2.03, p=.043$ (18 ${ }^{\circ}$ angle) $z=-6.07, p<.001$ ( $9^{\circ}$ angle). Although the decrease was smaller in the preterm group, $z=2.24$, $p=.025$, this group was less accurate at all levels of difficulty, $t(77)=-2.98, p=.004 ; t(85)=2.92, p=.005 ; t(105)=2.79, p=$ .006 , for angles of $27^{\circ}, 18^{\circ}$, and $9^{\circ}$, respectively. No other interactions were significant. Accuracy increased as VIQ and PIQ increased, $z=$ $2.45, p=.014$, at Step 2a, and, $z=2.51, p=.012$, at Step 2b. In each case, the contribution of group remained significant $(p=.008, p=$ .002 , respectively). The interactions between VIQ, PIQ, and group were not significant $(p>.05)$.

After controlling for the key covariates, responses in the preterm group were significantly slower, $F(1,107)=7.60, p=.007$. RTs increased in both groups as difficulty increased, $F(1,185)=$ 133.37, $p<.001$. A significant Group $\times$ Age interaction indicated that RTs in the full-term groups decreased significantly more with age, $F(1,108)=7.89, p=.006$. At Step 2a, VIQ did not contribute to RTs ( $p=.73$ ). At Step 2b, RTs decreased significantly as PIQ increased, $F(1,109)=8.35, p=.005$, but the contribution of group remained significant $(p=01)$. The interactions between VIQ, PIQ, and group were not significant $(p>.05)$.

\section{Matching Patterns}

After controlling for the key covariates, the preterm group was significantly less accurate than the control group, $z=-3.40, p=$ .001 . Neither VIQ nor PIQ contributed significantly to accuracy $(p>.05)$. The interactions between VIQ, PIQ, and group were not significant $(p>.05)$.

After controlling for the key covariates, the preterm groups responded more slowly, $F(1,105)=4.86, p<.03$. At Step 2a, VIQ did not contribute significantly to RTs $(p=.12)$. At Step $2 b$, RTs decreased significantly as PIQ increased, $F(1,109)=9.14$, $p=.003$. The contribution of group dropped below significance, $F(1,105)=2.26, p=.14$. No interactions were significant $(p>$ $.05)$. Regressing PIQ on group showed a significant negative association, $t=-13.59, p<.001$. This meets Baron and Kenny's (1986) conditions for mediation: the higher RTs in the preterm group could be explained by differences in the skill in and speed of the higher-level visuospatial analytical skills tapped by the PIQ subtests.

\section{Incomplete Patterns}

After controlling for the key covariates, the preterm group was significantly less accurate than the control group, $z=-2.93, p=$ .003. At Step 2a, accuracy increased significantly as VIQ increased, $z=2.84, p=.004$. The contribution of group dropped below significance $(p=.268)$. Regressing VIQ on group showed a significant negative association, $t(213)=-9.52, p<.001$. This meets Baron and Kenny's (1986) conditions for mediation: the lower accuracy in the preterm group could be explained by differ- ences in the skills tapped by the VIQ tasks. At Step 2b, accuracy increased significantly as PIQ increased, $z=2.79, p=.005$. The contribution of group decreased but remained significant ( $p=$ .038). The interactions between VIQ, PIQ, and group were not significant $(p>.05)$.

After controlling for the key covariates, the preterm group's responses were significantly slower, $F(1,108)=4.25, p=.04$. A significant Group $\times$ Age interaction indicated that RTs in the full-term group decreased significantly more rapidly with age, $F(1$, $108)=4.61, p=.03$. At Step 2a, RTs decreased marginally as VIQ increased, $F(1,107)=2.89, p=.09$. At Step $2 \mathrm{~b}$, RTs decreased significantly as PIQ increased, $F(1,108)=9.54, p=$ .003 . The contribution of group and the interaction between group and age dropped just below significance $(p=.063, p=.065$, respectively). Regressing PIQ on group showed a significant association, $b=-8.780, p<.001$, meeting the conditions for mediation, and indicating that, as with matching patterns, intergroup difference in performance could be explained by differences in the skill in and speed of the higher-level visuospatial analytical skills tapped by the PIQ subtests. The interactions between VIQ, PIQ, and group were not significant $(p>.05)$.

\section{Discussion}

Using task formats designed to reduce demands on attention, and controlling for the possibly confounding factors parental education and intellectual skill, we found that children born preterm responded more slowly and/or less accurately on four tasks tapping different types of visuospatial perception. These findings and their implications for the existence of specific visuospatial perceptual deficits in children born preterm will be discussed.

\section{Visuospatial-Processing Deficits}

The children born preterm performed less accurately on three of the four tasks. Consistent with research with very preterm born children, they made fewer correct responses when matching line orientations (Breslau et al., 1996; Luoma et al., 1998), matching patterns, and incomplete patterns (Davis et al., 2005; Hård et al., 2000; McGrath \& Sullivan, 2002). The absence of a difference on the remaining task, judging distance, likely reflects a ceiling effect: mean percentage of correct responses in both groups was above 98\%. The children born preterm also responded more slowly on all tasks. However, characteristics other than visuospatial perceptual skill may have affected their performance. As a group, children born preterm perform more poorly on measures of intelligence and motor skill and have more difficulty controlling attention (Mulder et al., 2009; Pizzo et al., 2010). Their family backgrounds may also equip them less well to manage the testing situation (Laucht et al., 1997). We consider these factors in turn.

Higher PIQ scores were associated with greater accuracy on three tasks and higher response speed on all tasks. PIQ mediated the association between performance and birth group on both matching patterns and incomplete patterns. This strong, consistent contribution was expected. PIQ reflects both skill in and speed of visuospatial perceptual analysis, and PIQ has been found to be associated with visuospatial perception in other studies, which used similar items (Davis et al., 2005; O'Reilly et al., 2010; Stiers et al., 1999). VIQ contributed most strongly to incomplete pat- 
terns, the most cognitively demanding task, where it mediated the association between birth status group and accuracy, and was marginally associated with response speed. This may reflect a tendency in both groups to use verbal mediation when representations of combinations of familiar shapes had to be formed and analyzed. In support of this, VIQ has been found to contribute to accuracy of visuospatial perception in other studies using similar items (Davis et al., 2005; O'Reilly et al., 2010). However, higher VIQ scores were also associated with greater accuracy on distance and higher response speed on line orientation, tasks that provide little basis for verbal mediation. Their association with VIQ may have reflected shared factors, such as motivation and social skill, which contribute more generally to performance. Despite the pervasive effect of intelligence, on three of the four tasks, the differences in accuracy, response speed, or both remained significant after intelligence had been taken into account.

Many children born preterm have significantly poorer motor skills than their full-term age-mates. This should not have affected accuracy of responding, where deficits were found on three tasks. Although poorer motor skills may have contributed to the differences in RTs found on all tasks, we find it unlikely that they completely explain them. The same movement was required for each item on each task. If the differences in RTs were based purely on differences in motor skill, they should have remained rather constant over the difficulty conditions within a task and over the tasks themselves. Instead, they changed significantly as distance and line orientation became more difficult, and they ranged from less than $200 \mathrm{~ms}$ on the easiest conditions of the elementary tasks to $820 \mathrm{~ms}$ on the higher-level tasks. Background factors associated with parental education contributed to speed of performance on three tasks. However, controlling for parental education also failed to eliminate group differences. Differences in speed-accuracy trade-off can also be ruled out because children born preterm responded both more slowly and less accurately. Given these considerations, and task formats designed to reduce the impact of poor attentional control, we consider that these findings provide robust evidence for the existence of visuospatial perceptual deficits in children born preterm. The Age $\times$ Group interactions were significant on two tasks, reflecting a smaller decrease in the preterms' RTs with age. This suggests that they had not "grown out" of their deficits at 11 years of age.

One interpretation of these findings is that they indicate a deficit in both dorsal and ventral stream functioning in the children born preterm. Significantly slower judgments of distance, which draw predominantly on dorsal stream functioning (Chen et al., 2000; Fias et al., 2002), suggest a dorsal deficit. This is consistent with the findings of previous studies (Atkinson \& Braddick, 2007; Foreman et al., 1997; Santos et al., 2010; Taylor et al., 2009) and with the well-established vulnerability of periventricular white matter following preterm birth (Volpe, 2009). However, these children were also slower on line orientation, where areas in the ventral extrastriate cortex are thought to limit speed of processing (Ng et al., 2001) and both slower and less accurate on matching patterns, a task thought to activate a broad network of areas including but not restricted to the dorsal pathway (Calhoun et al., 2001). This suggests that their visuospatial deficits are not limited to dorsal stream perceptual processes, a suggestion consistent with previous studies, which have found that children born preterm perform worse on tasks associated with the ventral stream, includ- ing the pen-and-pencil version of the TVPS (Davis et al., 2005), and with the widespread differences in brain structure found later in childhood in this group (Ment et al., 2009; Nosarti et al., 2002; Peterson et al., 2000; Volpe, 2009).

A second interpretation is that inefficient dorsal functioning in the children born preterm was the basis for their poorer performance on all tasks. Although behavioral and imaging studies have provided strong evidence for the existence of two distinct visual streams, in practice, even elementary visual perceptual functions involve the activity of several different areas of the brain, and are therefore unlikely to be mediated entirely by one or other visual stream (Creem \& Proffitt, 2001). Imaging and lesion studies of judgment of line orientation, for example, have shown that, while areas in the ventral extrastriate cortex play a distinctive role in simultaneous judgments of line orientation, areas in the posterior parietal lobe are also involved (Dupont et al., 1998; Isaacs et al., 2003). The processing of novel combinations of familiar visual stimuli, called on by matching patterns, places high demands on spatial attention, which is mediated by the posterior parietal cortex (Cohen et al., 2008). Inefficient dorsal functioning may then have lowered the performance of children born preterm on line orientation and matching patterns. Dorsal and ventral streams are involved with a range of visuospatial perceptual functions, which differ in nature and complexity. Further research using tasks designed to tap different aspects and levels of dorsal and ventral functioning is needed to determine more precisely how the dorsal and ventral streams contribute to the visuospatial perceptual problems of children born preterm. Tasks tapping functions in the different streams at similar levels of complexity are particularly informative (see Santos et al., 2010, for an example).

\section{Study Limitations}

The dorsal and ventral streams are involved in a range of visuospatial functions. The dorsal stream is also involved in spatial attention. Our tasks did not allow us to determine the basis for the visuospatial perceptual deficits we identified. Investigations using tasks, which tap specific dorsal and ventral functions, and which separate out perceptual and attentional functions of the dorsal stream, would help identify the aspects of visuospatial perception, which are most impaired following preterm birth.

The results are also based on the performance of a small group of children from a single medical center with no serious neurological or motor problems. None of the children was in the most vulnerable group of preterms (GAs between 23 and 24 weeks) while six were born at GAs between 32 and 34 weeks. This raises the question how well they can be generalized to the broader population of children born preterm. The below average mean IQ scores, and above average frequencies of behavior problems, in the participants were highly similar to those found in other follow-up studies of children born preterm in the last decade of the last century (Bhutta et al., 2002). Although this increases our confidence that these results can be generalized to the broader population of children born preterm in this period who did not go on to develop serious neurological problems, investigations of children from other centers are needed to describe more precisely the visuospatial perceptual problems that seem to be more frequent in this group. 


\section{Conclusion}

This is the first study, to our knowledge, to investigate both the speed and the accuracy of visuospatial perception in children born very preterm, and to control for the influence of intelligence and parental education. We found convincing evidence of pervasive visuospatial perceptual deficits, adding to the existing evidence for a more general deficit in visuospatial processing in this group. Lower intelligence scores and parental education levels added to the effect of birth status to produce substantial deficits in the preterm group. These deficits are likely to be associated with poorer performance on daily life tasks, which demand rapid, accurate visuospatial perception, for example, learning to recognize letters, words, and the quantitative relationships underlying mathematical concepts. Future research teasing apart the role of dorsal and ventral perceptual impairments is needed to describe visuospatial perceptual deficits more precisely and to identify ways of limiting their impact on daily life activities.

\section{References}

Atkinson, J., \& Braddick, O. (2007). Visual and visuocognitive development in children born very prematurely. Progress in Brain Research, 164, 123-149. doi:10.1016/S0079-6123(07)64007-2

Baron, R. M., \& Kenny, D. A. (1986). The moderator-mediator variable distinction in social psychological research: Conceptual, strategic, and statistical considerations. Journal of Personality and Social Psychology, 51, 1173-1182. doi:10.1037/0022-3514.51.6.1173

Benton, A. L., Hamsher, K. de, S., Varney, N. R., \& Spreen, O. (1983). Judgment of line orientation, form $\mathrm{H}$ in contributions to neuropsychological assessment. New York, NY: Oxford University Press.

Bhutta, A. T., Cleves, M. A., Casey, P. H., Cradock, M. M., \& Anand, K. J. S. (2002). Cognitive and behavioural outcomes of school-aged children who were born preterm: A meta analysis. JAMA: Journal of the American Medical Association, 288, 728-737. doi:10.1001/ jama.288.6.728

Brazy, J. E., Eckerman, C. O., Oehler, J. M., Goldstein, R. F., \& O'Rand, A. M. (1991). Nursery neurobiologic risk score: Important factors in predicting outcome in very low birth weight infants. Journal of Pediatrics, 118, 783-792.

Breslau, N., Chilcoat, H., DelDotto, J., \& Andreski, P. (1996). Low birth weight and neurocognitive status at six years of age. Biological Psychiatry, 40, 389-397. doi:10.1016/0006-3223(95)00399-1

Calhoun, V. D., Adali, T., McGinty, V. B., Pekar, J. J., Watson, T. D., \& Pearlson, G. D. (2001). fMRI activation in a visual-perception task: Network of areas detected using the general linear model and independent components analysis. NeuroImage, 14, 1080-1088. doi:10.1006/ nimg.2001.0921

Caravale, B., Tozzi, C., Albino, G., \& Vicari, S. (2005). Cognitive development in low risk preterm infants at 3-4 years of life. Archives of Disease in Childhood Fetal and Neonatal Edition, 90, F474-9. doi: 10.1136/adc.2004.070284

Chen, J., Myerson, J., Hale, S., \& Simon, A. (2000). Behavioral evidence for brain-based ability factors in visuospatial information processing. Neuropsychologia, 38, 380-387. doi:10.1016/S0028-3932(99)00095-0

Cohen, L., Dehaene, S., Vinckier, F., Jobert, A., \& Montavont, A. (2008). Reading normal and degraded words, contribution of the dorsal and ventral visual pathways. NeuroImage, 40, 353-366. doi:10.1016/ j.neuroimage.2007.11.036

Constable, R. T., Ment, L. R., Vohr, B. R., Kesler, S. R., Fulbright, R. K., Lacadie, C., \& Reiss, A. R. (2008). Prematurely born children demonstrate white matter microstructural differences at 12 years of age, relative to term control subjects: An investigation of group and gender effects. Pediatrics, 121, 306-316. doi:10.1542/peds.2007-0414
Creem, S. H., \& Proffitt, D. R. (2001). Defining the cortical visual systems: 'What', 'Where', and 'How'. Acta Psychologica, 107, 43-68. doi: 10.1016/S0001-6918(01)00021-X

Davis, D. W., Burns, B. M., Wilkerson, S. A., \& Steichen, J. J. (2005). Visual perceptual skills in children born with very low birth weights. Journal of Pediatric Health Care, 19, 363-368. doi:10.1016/ j.pedhc.2005.06.005

de Vries, L. S., Eken, P., \& Dubowitz, L. M. S. (1992). The spectrum of leukomalacia using cranial ultrasound. Behavioural Brain Research, 49 , $1-6$.

Dupont, P., Vogels, R., Vandenberghe, R., Rosier, A., Cornette, L., Bormans, G., \& Orban, G. A. (1998). Regions in the human brain activated by simultaneous orientation discrimination: A study with positron emission tomography. European Journal of Neuroscience, 10, 3689-3699.

Eysenck, M. W., Derakshan, N., Santos, R., \& Calvo, M. G. (2007). Anxiety and cognitive performance: Attentional control theory. Emotion, 7, 336-353. doi:10.1037/1528-3542.7.2.336

Fias, W., Dupont, P., Reynvoet, B., \& Orban, G. A. (2002). The quantitative nature of a visual task differentiates between ventral and dorsal stream. Journal of Cognitive Neuroscience, 14, 646-658. doi:10.1162/ 08989290260045873

Foreman, N., Fielder, A., Minshell, C., \& Hurrion, E. (1997). Visual search, perception, and visual-motor skill in 'healthy' children born at 27-32 weeks' gestation. Journal of Experimental Child Psychology, 64, 27-41. doi:10.1006/jecp.1996.2326

Gardner, M. F. (1996). Test of Visual-Perceptual Skills (Non-Motor) Revised. Hydesville, CA: Psychological and Educational Publications.

Giménez, M., Junqué, C., Narberhaus, A., Bargalló, N., Botet, F., \& Mercader, J. M. (2006). White matter volume and concentration reductions in adolescents with history of very preterm birth: A voxel-based morphometry study. NeuroImage, 32, 1485-1498. doi:10.1016/ j.neuroimage.2006.05.013

Goyen, T. A., Lui, K., \& Woods, R. (1998). Visual-motor, visualperceptual, and fine motor outcomes in very-low birthweight children at 5 years. Developmental Medicine \& Child Neurology, 40, 76-81. doi: 10.1111/j.1469-8749.1998.tb15365.x

Gozzo, Y., Vohr, B., Lacadie, C., Hampson, M., Katz, K. H., MallerKesselman, J., \& Ment, L. R. (2009). Alterations in neural connectivity in preterm children at school age. NeuroImage, 48, 458-463. doi: 10.1016/j.neuroimage.2009.06.046

Hård, A., Niklasson, A., Svensson, E., \& Hellström, A. (2000). Visual function in school-aged children born before 29 weeks of gestation: A population-based study. Developmental Medicine \& Child Neurology, 42, 100-105. doi:10.1017/S0012162200000207

Inder, T. E., Warfield, S. K., Wang, H., Hüppi, P. S., \& Volpe, J. J. (2005). Abnormal cerebral structure is present at term in premature infants. Pediatrics, 115, 286-294. doi:10.1542/peds.2004-0326

Isaacs, E. B., Edmonds, C. J., Chong, W. K., Lucas, A., \& Gadian, D. G. (2003). Cortical anomalies associated with visuospatial processing deficits. Annals of Neurology, 53, 768-773. doi:10.1002/ana.10546

Isaacs, E. B., Lucas, A., Chong, W. K., Wood, S. J., Johnson, C. L., \& Marshall, C. (2000). Hippocampal volume and everyday memory in children of very low birth weight. Pediatric Research, 47, 713-720. doi:10.1203/00006450-200006000-00006

Jakobson, L. S., Frisk, V., \& Downie, A. L. S. (2006). Motion-defined form processing in extremely premature children. Neuropsychologia, 44 1777-1786. doi:10.1016/j.neuropsychologia.2006.03.011

Jakobson, L. S., Frisk, V., Knight, R. M., Downie, A. L. S., \& Whyte, H. (2001). The relationship between periventricular brain injury and deficits in visual processing among extremely-low-birthweight (1000 g) children. Journal of Pediatric Psychology, 26, 503-512. doi:10.1093/ jpepsy/26.8.503

Kesler, S. R., Reiss, A. L., Vohr, B., Watson, C., Schneider, K. C., Katz, K. H., \& Ment, L. R. (2008). Brain volume reductions within multiple 
cognitive systems in male preterm children at age twelve. The Journal of Pediatrics, 152, 513-520. doi:10.1016/j.jpeds.2007.08.009

Klein, N. K. (1988). Children who were very low birthweight: Cognitive abilities and classroom behavior at five years of age. The Journal of Special Education, 22, 41-54. doi:10.1177/002246698802200107

Kloosterman, G. J. (1970). On intrauterine growth. The significance of prenatal care. International Journal of Gynaecological Obstetrics, 8, 895-912.

Kort, W., Compaan, E. L., Bleichrodt, N., Resing, W. C. M., Schittekatte, M., Bosmans, M., \& Verhaeghe, P. (2002). WISC-III ${ }^{N L}$ : Wechsler Intelligence Scales for Children (3rd ed., Dutch Version). Amsterdam, Netherlands: NIP Dienstencentrum.

Laucht, M., Esser, G., \& Schmidt, M. H. (1997). Developmental outcome of infants born with biological and psychosocial risks. Journal of Child Psychology and Psychiatry, 38, 843-853. doi:10.1111/j.14697610.1997.tb01602.x

Luoma, L., Herrgård, E., \& Martikainen, A. (1998). Neuropsychological analysis of the visuomotor problems in children born preterm at $\leq 32$ weeks of gestation: A 5-year prospective follow-up. Developmental Medicine \& Child Neurology, 40, 21-30. doi:10.1111/j.14698749.1998.tb15352.x

McGrath, M., \& Sullivan, M. (2002). Birth weight, neonatal morbidities, and school age outcomes in full-term and preterm infants. Issues in Comprehensive Pediatric Nursing, 25, 231-254. doi:10.1080/ 01460860290042611

Ment, L. R., Hirtz, D., \& Hüppi, P. S. (2009). Imaging biomarkers of outcome in the developing preterm brain. Lancet Neurology, 8, 10421055. doi:10.1016/S1474-4422(09)70257-1

Milner, A. D., \& Goodale, M. A. (2006). The visual brain in action. Oxford, England: Oxford University Press.

Mishkin, M., Ungerleider, L. G., \& Macko, K. A. (1983). Object vision and spatial vision: Two cortical pathways. Trends in Neurosciences, 6, 414-417. doi:10.1016/0166-2236(83)90190-X

Mulder, H., Pitchford, N. J., Hagger, M., \& Marlow, N. (2009). Development of executive function and attention in preterm children: A systematic review. Developmental Neuropsychology, 34, 393-421. doi: $10.1080 / 87565640902964524$

Ng, V. W. K., Bullmore, E. T., de Zubicaray, G. I., Cooper, A., Suckling, J., \& Williams, S. C. R. (2001). Identifying rate-limiting nodes in large-scale cortical networks for visuospatial processing: An illustration using fMRI. Journal of Cognitive Neuroscience, 13, 537-545. doi: $10.1162 / 08989290152001943$

Ng, V. W. K., Eslinger, P. J., Williams, S. C. R., Brammer, M. J., Bullmore, E. T., Andrew, C. M., \& Benton, A. L. (2000). Hemispheric preference in visuospatial processing: A complementary approach with fMRI and lesion studies. Human Brain Mapping, 10, 80-86. doi: 10.1002/(SICI)1097-0193(200006)10:2<80::AID-HBM40>3.0.CO;2-2

Nosarti, C., Al-Asady, M. H. S., Frangou, S., Stewart, A. L., Rifkin, L., \& Murray, R. M. (2002). Adolescents who were born very preterm have decreased brain volumes. Brain, 125, 1616-1623. doi:10.1093/brain/ awf157

O'Reilly, M., Vollmer, B., Vargha-Khadem, F., Neville, B., Connelly, A., Wyatt, J., \& de Haan, M. (2010). Ophthalmological, cognitive, electrophysiological and MRI assessment of visual processing in preterm children without major neuromotor impairment. Developmental Science, 13, 692-705. doi:10.1111/j.1467-7687.2009.00925.x

Papile, L., Burstein, J., Burstein, R., \& Koffler, H. (1978). Incidence and evolution of subependymal and intraventricular hemorrhage: A study of infants with birth weights less than $1500 \mathrm{gm}$. Journal of Pediatrics, 92, 529-534.

Peterson, B. S., Vohr, B., Staib, L. H., Cannistraci, C. J., Dolberg, A., Schneider, K. C., \& Ment, L. R. (2000). Regional brain volume abnormalities and long-term cognitive outcome in preterm infants. JAMA: Journal of the American Medical Association, 284, 1939-1947. doi: 10.1001/jama.284.15.1939

Pizzo, R., Urban, S., Van Der Linden, M., Borradori-Tolsa, C., Freschi, M., Forcada-Guex, M., \& Barisnikov, K. (2010). Attentional networks efficiency in preterm children. Journal of the International Neuropsychological Society, 16, 130-137. doi:10.1017/S1355617709991032

Santos, A., Duret, M., Mancini, J., Busuttil, M., \& Deruelle, C. (2010). Does preterm birth affect global and configural processing differently? Developmental Medicine \& Child Neurology, 52, 293-298. doi:10.1111/ j.1469-8749.2009.03398.x

Sattler, J. M. (1992). Assessment of children. San Diego, CA: Jerome M. Sattler.

Smits-Engelsman, B. C. M. (1998). The Movement Assessment Battery for Children - Dutch edition. Lisse. Netherlands: Swets and Zeitlinger.

Stiers, P., De Cock, P., \& Vandenbussche, E. (1999). Separating visual perception and non-verbal intelligence in children with early brain injury. Brain \& Development, 21, 397-406. doi:10.1016/S03877604(99)00050-9

Taylor, H. G., Minich, N. M., Klein, N., \& Hack, M. (2004). Longitudinal outcomes of very low birth weight, Neuropsychological findings. Journal of the International Neuropsychological Society, 10, 149-163.

Taylor, N. M., Jakobson, L. S., Maurer, D., \& Lewis, T. L. (2009). Differential vulnerability of global motion, global form, and biological motion processing in full-term and preterm children. Neuropsychologia, 47, 2766-2778. doi:10.1016/j.neuropsychologia.2009.06.001

Teplin, S. W., Burchinal, M., Johnson-Martin, N., Humphry, R. A., \& Kraybill, E. N. (1991). Neurodevelopmental, health, and growth status at age 6 years of children with birth weights less than 1001 grams. Journal of Pediatrics, 118, 768-777. doi:10.1016/S0022-3476(05)80045-9

Tibber, M. S., Anderson, E. J., Melmoth, D. R., Rees, G., \& Morgan, M. J. (2009). Common cortical loci are activated during visuospatial interpolation and orientation discrimination judgements. PLoS One, 4, e4585. doi:10.1371/journal.pone.0004585

Trojano, L., Fragassi, N. A., Chiacchio, L., Izzo, O., Izzo, G., Di Cesare, G., \& Grossi, D. (2004). Relationships between constructional and visuospatial abilities in normal subjects and in focal brain-damaged patients. Journal of Clinical and Experimental Neuropsychology, 26, 1103-1112. doi:10.1080/13803390490515522

Vicari, S., Caravale, B., Carlesimo, G. A., Casadei, A. M., \& Allemand, F. (2004). Spatial working memory deficits in children at ages 3-4 who were low birth weight, preterm infants. Neuropsychology, 18, 673-678. doi:10.1037/0894-4105.18.4.673

Volpe, J. J. (2009). Brain injury in premature infants: A complex amalgam of destructive and developmental disturbances. Lancet Neurology, 8, 110-124. doi:10.1016/S1474-4422(08)70294-1

Zaehle, T., Jordan, K., Wüstenberg, T., Baudewig, J., Dechent, P., \& Mast, F. W. (2007). The neural basis of the egocentric and allocentric spatial frame of reference. Brain Research, 1137, 92-103. doi:10.1016/ j.brainres.2006.12.044

Received September 9, 2011

Revision received May 7, 2012

Accepted May 21, 2012 